\title{
Research on Simulation Playback System of Vehicle Trajectory
}

\author{
Shuang Shi ${ }^{1, *}$, Yanmei Wang ${ }^{1}$, Fei Peng ${ }^{1}$ and Daqi Zhang ${ }^{2}$ \\ ${ }^{1}$ Anhui Sanlian University, Hefei, China \\ ${ }^{2}$ Qinghai University, Xining, China \\ ${ }^{*}$ Corresponding author
}

\begin{abstract}
In order to facilitate learning, increase reliability, we take advantage of the video playback system in the subjects second and third of driving exam. Vehicle trajectory can be replayed with small amount of data transferred or stored up. The system is composed of four parts: the Beidou navigation system, the Beidou positioning and receiving device, the wireless communication network and the upper computer. The positioning information of the vehicle is received by the Beidou positioning device, and it is transmitted to the host computer through the wireless communication network. The upper computer inputs the information into the simulation animation which is displayed in real time. The results of the study are of great practical value.
\end{abstract}

\section{Keywords—driving exam; simulation; Beidou}

\section{INTRODUCTION}

With the increase of car population, the number of joining driving exam has also multiplied. It has become the basic requirement of modern people that mastering the driving technology and driving license. There are three main methods for the study and examination system of traditional subjects $2^{\text {th }}$ and $3^{\text {th }}$. The first is to adopt PC, infrared sensor and vibration sensor ${ }^{[1][2]}$; The second is the use of $\mathrm{PC}$ and hall sensors ${ }^{[3]}$; The third is the high-precision evaluation system of intelligent vehicle driving based on differential positioning of $\mathrm{GNSS}^{[4]}$. These methods have a low rate. large number of staff have been backlogged. It leads to a decline in the quality of examinations, which indirectly affects the traffic safety ${ }^{[5]}$. After many years of development, the driving exam product has been developed from the early artificial mode to the present machine-aided mode. At present, the mainstream approach is to use Beidou positioning combined with inertial measurement. The vehicle's information of position, posture and speed is measured high accurately, and the information is transferred through the network to exam center. Test project is evaluated automatically. The problem of the exam is that transparency is not high enough. When examinees want to check their error status, they can only see through video playback. But the coverage of video is not high in some exam areas, and it is influenced by shooting Angle, which causes video to be restricted. At the same time, video data has large amount of storage space. We can solve this problem by using video simulation playback technology. The technology of video Simulation playback had been applied widely, such as the "eagle eye" in sports of badminton, tennis. it was achieved by "3D" simulation with using multiple cameras data.

\section{SYSTEM CHARACTERISTICS}

Trajectory simulation playback of exam vehicle is achieved with the Beidou positioning data. According to the data of multiple positioning receivers, the positioning of road, drill ground and vehicle is calculated. According to the difference data type, pseudo distance of difference is about $10 \mathrm{~cm}$, while the accuracy of carrier phase is $1 \mathrm{~cm}^{[6-8]}$. Those provide the data support for the video simulation playback of exam vehicle. Combined the wireless network communication technology, the video simulation playback can be realized.

The characteristics of the system are as follows:

(1) No need to increase the equipment. It can be realized by using the existing positioning system of exam vehicle.

(2) The amount of data transmission is small. The trajectory playback can be achieved by transferring the positioning information to the upper computer.

(3) Avoiding falsification and improving the reliability of the examination. Because of the small amount of data, it can be passed easily to the Internet. Examinees can check their trajectory on the Internet at any time, and at the same time they can also observe others' achievement, so they can learn and improve easily.

(4) It can overcome the limits and blind areas of human observation, and help examinees and examiners make accurate judgments.

\section{GENERAL IDEA}

The system consists of four parts: the Beidou navigation system, the Beidou positioning and receiving device, the wireless communication network and upper computer. The positioning data of the Beidou navigation is received by the Beidou positioning receiver. The data contains positioning information of road, drill ground and vehicle. The examination information is ground coordinate of road, drill ground, such as coordinate of reference point on "S" road, wheeling in right angle, road of subjects $3^{\text {th }}$. The coordinate is relatively fixed, so we calibrate it only once in general; Information of exam vehicle is obtained by the receiving device that is mounted on vehicle. The device can obtain real-time coordinates of vehicle. The information is transmitted to the upper computer via the wireless communication network. The upper computer inputs the coordinate information into the simulation animation, and stores it or displays it in real time. The system architecture is shown in Figure 1: 


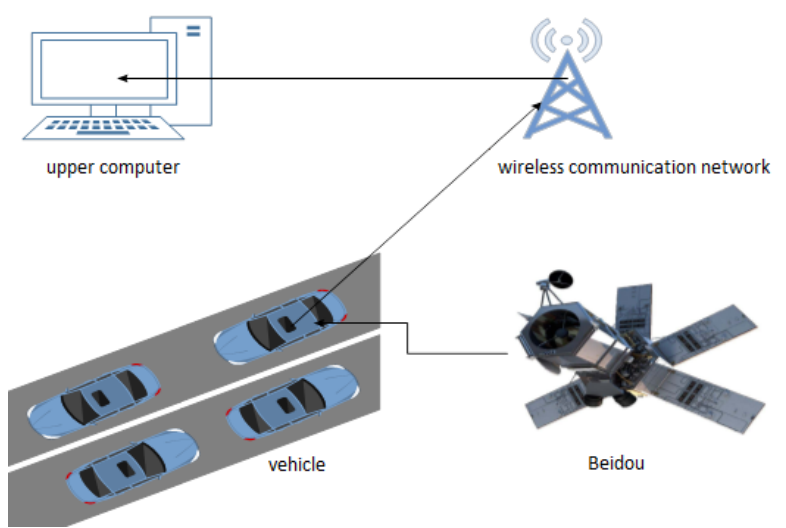

FIGURE I. SYSTEM ARCHITECTURE

\section{SOFTWARE DESIGN}

The software is mainly placed in the upper computer. The main contents of computer program include GPRS communication protocol, data format of Beidou system, software framework of main program and embedded system. The key design includes two aspects: data correction and direction prediction.

\section{A. Data Correction}

If we want to get the accurate contour data, we need to measure the movement posture in real time, and correct data of attitude change in real time.

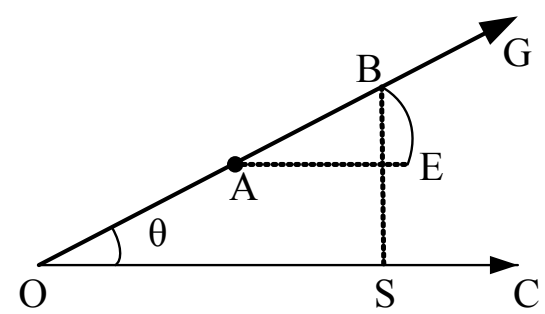

FIGURE II. DATA CORRECTION

As shown in Figure 2. OC is a horizontal plane and OG is a ramp. When the vehicle enters the ramp, the vehicle positioning is in the A point. The distance between positioning system and head of vehicle is $\mathrm{L}_{\mathrm{AB}}$. If the $\mathrm{B}$ point is the edge of the stop line, the vehicle just missed the line. but in the GPS' data, the $\mathrm{B}$ point is at the projection position of $\mathrm{S}$ point. If the trajectory simulation data does not be corrected, the head of vehicle will be located at the $\mathrm{E}$ point. In this way, because the $\mathrm{E}$ point is beyond the limit, it will be misjudged to be a collision line. The correction process is as follows:

Supposing, distance after corrected is $L_{A B}^{\prime}$ between $A$ and head of vehicle.

$$
L_{\mathrm{AB}}=L_{A B} \times \cos \theta
$$

\section{B. Direction Prediction of Exam Vehicle}

The positioning system is installed in a certain position of the vehicle. Because of the changes of drill ground of exam and the attitude of exam vehicle, system will be insensitive to the position change of body which is far away from system. When the vehicle turns to the direction, the position of the positioning device will lag behind the position change of head or rear of the vehicle. In order to keep the image consistent with the actual vehicle in simulation, it is necessary to predict the position of the vehicle. The method of curve fitting is used. According to the principle of vehicle variation, the circle function is used to predict. The function is as follows:

$$
x^{2}+y^{2}=a^{2} \quad(x, y \geq 0)
$$

The algorithm flow is shown in Figure 3.

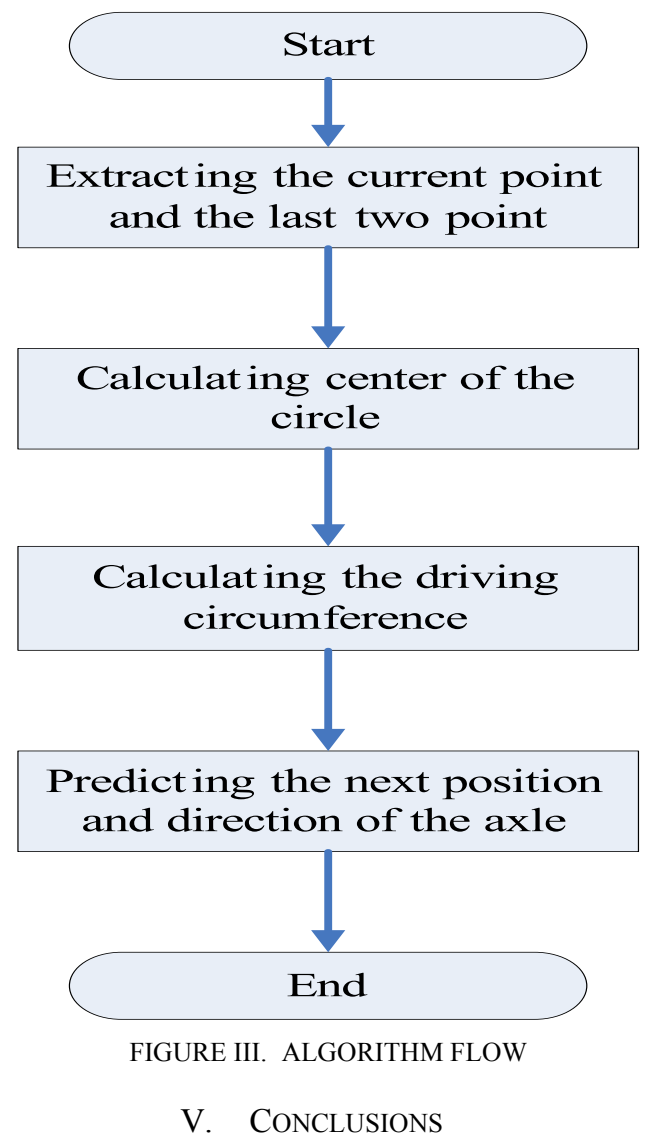

The technology of simulation playback is applied to tracking of the vehicle trajectory in driving exam. we used less amount of data to achieve simulation video playback. The problem of shortage of bandwidth and large amount of data was solved. Positioning data through the hardware platform was transferred in real-time, and it ensures the real-time monitoring to vehicle and related personnel to view easily.

\section{ACKNOWLEDGMENTS}

This research is supported by the fund of Traffic Safety Application Technology Innovation Center of Anhui Sanlian 
University(No.YJT17002) and National Nature Science Foundation of China(No.51769027).

\section{REFERENCES}

[1] Jun Wu. Research and design of intelligent driver field examination system [D]. Xidian University,2010.

[2] [2] Xiaoqiong Dong. Research on Application of infrared sensor [J]. The Science and Education Article Collects, 2006,02:161-162.

[3] Jun Guo, Heping Liu, Ping Liu. Application of hall sensor based on high current detection[J]. Transducer and Microsystem Technologies,2011,05:142-145.

[4] Yubao Chen, Tao An, Heng Hu, Jianyong Li, Yu Chun Gao. Research on accurate positioning of weather radar coordinates based on GNSS differential positioning [J]. Meteorological Monthly, 2013,03:389-393.

[5] Pengrui Bai, Song Gao, The existing problems of motor vehicle driving training examination and the application of electronic examination system [J]. Electronic Test,2013,14:81-82.

[6] Jie Wang, Guannan Xu. Research and Discussion on relative positioning method based on GNSS dynamic difference [J]. Modern Navigation, 2015,03:250-256.

[7] Bao Li, Fei Wang, Yinbing Zhu, Yanfeng Hu. Performance comparison and analysis of different positioning modes of GNSS [J]. Ship Electronic Engineering,2013,06:68-71.

[8] Xiaodong Wu, Gang Shen, Wei Xia, Hao Li, Tao Li, Yue Xie, JingLi. Research on GNSS differential information broadcasting technology in traffic industry [J]. Gnss World of China,2015,01:41-45. 